\title{
Association between serum adiponectin levels with gestational diabetes mellitus and postpartum metabolic syndrome:A case control study
}

\author{
Zoleikha Atarod ${ }^{1}$, Mostafa Ebrahemian ${ }^{2}$, Hamed Jafarpour ${ }^{3}$, Mahta Moraghebi ${ }^{4}$, \\ Elham SHARAFKHANI ${ }^{5}$
}

\begin{abstract}
${ }^{1}$ Department of Obstetrics and Gynecology, School of Medicine, Mazandaran University of Medical Sciences, Sari, Iran; ${ }^{2}$ Resident of Anaesthesiology, Mazandaran University of Medical Science, Sari, Iran; ${ }^{3}$ Student Research Committee, Mazandaran University of Medical Science, Sari, Iran; ${ }^{4}$ Student Research Committee, Hormozgan University of Medical Science, Bandar Abbas, Iran; ${ }^{5}$ Resident of Obstetrics \& Gynaecology, Mazandaran University of Medical Science, Sari, Iran E-mail: Elham.sharafkhani@gmail.com
\end{abstract}

Objective. Pregnancy can cause diabetic conditions and gestational diabetes is the most common metabolic disorder of the era. Scientific evidence suggests that obesity increases the incidence and severity of gestational diabetes. Adipokines are proteins secreted from adipose tissue in response to extracellular stimuli and altered metabolism. These hormones are involved in regulating the energy balance, lipid metabolism, and insulin sensitivity. One of the types of adipokines is called adiponectin, which has anti-diabetic, anti-inflammatory, and anti-atherogenic effects. Accordingly, this study is aimed to investigate the correlation between the serum adiponectin level with the gestational diabetes and the postpartum metabolic syndrome.

Methods. This case-control study was carried out on 37 pregnant women (in Sari, Iran) with gestational diabetes and 37 non-diabetic pregnant women who were matched regarding age and body mass index (BMI). Serum adiponectin and glucose levels were measured. Finally, six weeks after termination of pregnancy, women in both groups were evaluated for metabolic syndrome. All statistical analyses of this study were performed using IBM SPSS software version 21 and, in all cases, the two-way $\mathrm{p}$ value less than 0.05 was considered statistically significant.

Results. The mean age of pregnant women was $28.46 \pm 4.11$ years in the non-diabetic group and $30.03 \pm 4.71$ in the diabetic group. There was no statistically significant difference found between the mean age $(\mathrm{p}=0.123)$ and $\mathrm{BMI}(\mathrm{p}=0.727)$ in two groups. Serum adiponectin levels in the diabetic group $(5.51 \pm 3.15 \mu \mathrm{g} / \mathrm{ml})$ were significantly lower than in the non-diabetic group $(8.35 \pm 4.54 \mu \mathrm{g} / \mathrm{ml})$ $(\mathrm{p}=0.003)$. In the diabetic group, serum adiponectin level did not correlate with the maternal age, maternal BMI, and postpartum metabolic syndrome $(\mathrm{p}>0.005)$.

Conclusions. The results of the present study indicate a correlation of low adiponectin concentrations with gestational diabetes, but this association with postpartum metabolic syndrome is uncertain. However, to elucidate the mechanism of adiponectin in predicting gestational diabetes and postpartum metabolic syndrome further studies are required.

Key words: adiponectin, gestational diabetes, metabolic syndrome

Pregnancy is accompanied by many anatomical and physiological changes. Healthy pregnancy requires metabolic as well as hormonal adaptations that involve the hypothalamus, pituitary, parathy- roid, thyroid, and adrenal glands. This metabolic adaptation meets the needs of the fetus during pregnancy (Gonzales Gonzalez et al. 2014). Moreover, placental secretions including progesterone, 
placental lactogen, corticotropin-releasing hormone, and growth hormone, along with maternal hormonal changes, increase insulin resistance and insulin secretion, making pregnancy a diabetic condition and resulting in gestational diabetes as the most common metabolic disorder in this period (Maruotti et al. 2014). The prevalence of gestational diabetes has been reported to be between 1-14\% worldwide, while it is estimated to be $4.5 \%$ in Iran. The disease has many effects on the mother and fetus, the most common, of which are: macrosomia, birth defects, cesarean section, polyhydramnios, preeclampsia and neonatal metabolic disorders (hypoglycemia, hypercalcemia, hyperbilirubinemia), and finally long-term complications including maternal type II diabetes in the postpartum period (Miller et al. 2013; Khong et al. 2015).

Insulin resistance and deficiency in pancreatic beta cells is a characteristic of gestational diabetes, which on the one hand leads to an abnormal increase of glucose production by the liver and on the other hand reduces glucose consumption by adipose and muscle tissues. Cytokines such as tumor necrosis factor alpha (TNF- $\alpha$ ) and interleukine-6 (IL-6) are thought to increase insulin resistance and consequently hyperinsulinemia on one hand, and lead to increased placental leptin gene expression and decreased adiponectin gene expression on the other hand (Di Cianni et al. 2003). As a result, these factors exacerbate inflammation and decrease insulin sensitivity, while increasing insulin resistance in gestational diabetes (Khong et al. 2015).

Adiponectin is one of the most abundant adipose tissue-specific proteins that is expressed and secreted only in this tissue. Adiponectin has effects such as insulin sensitivity, prevention of vascular inflammation, and anti-atherogenic behavior (Ouchi et al. 2000). The results regarding serum adiponectin levels in pregnant women with gestational diabetes compared with healthy pregnant women are inconsistent. Several studies have reported low serum adiponectin levels in gestational diabetes (Fuglsang et al. 2006; Mastorakos et al. 2007; Mazaki-Tovi et al. 2007) and several studies also have shown no significant difference in serum adiponectin levels between gestational diabetes and healthy gestation. Few studies have been conducted on gestational diabetes, especially in Asian and Iranian populations regarding the adiponectin to nectin ratio as a new indicator of insulin resistance and its association with the wellknown insulin resistance index (HOMA-IR). One study has shown the effect of race on plasma concentrations of adiponectin during pregnancy that may be important in increasing risk of diabetes (Retnakaran et al. 2004). Therefore, the aim of this study was to investigate the relationship between serum adiponectin levels and gestational diabetes.

Decreased adiponectin levels may play a role in the pathogenesis of GDMs, and several studies have found that low adiponectin levels in pregnant women are associated with GDM (Hedderson et al. 2013). In addition to the unfavorable outcomes of gestational diabetes, a 5-year postpartum diabetes history increases the risk of type II diabetes by $18-50 \%$ (Metzger et al. 1993; Kaufmann et al. 1995). Recent studies have also shown that gestational diabetes increases the risk of hypertension and dyslipidemia resulting in long-term atherosclerosis and cardiovascular disease (Innes and Wimsatt 1999; Daviglus et al. 2004), which can be due to common risk factors in both diseases (Kim et al. 2002; Ben-Haroush et al. 2004).

Early diagnosis using early screening to identify and prevent type II diabetes in women with a history of gestational diabetes is a good opportunity to reduce the incidence and frequency of this disease (BentleyLewis et al. 2008). In this regard, studies have shown that women with impaired glucose tolerance or women with postpartum metabolic syndrome are at higher risk for developing diabetes (Strehlow and Mestman 2005). Accordingly, adiponectin assessment during pregnancy may be a useful indicator for understanding gestational diabetes and its association with postpartum metabolic syndrome indices. Therefore, the present study was designed to evaluate the correlation between adiponectin levels and postpartum metabolic syndrome.

\section{Subjects and Methods}

Subjects. This case-control study was performed on pregnant women at the age range of 20-35 years old, who had referred to maternal care clinic in Mostafavian clinic of Sari during July 2018 to February 2019. Sample size was considered to be $90(\mathrm{n}=90)$, of which 45 patients were randomly assigned to the case group and 45 to the control group, while both groups were also matched. Inclusion criteria consisted of pregnant women aged $20-35$ years with gestational age of 28-24 weeks, and exclusion criteria were smoking (any amount), rheumatic inflammatory diseases and vascular collagen (history of any of these diseases based on history obtained from the patient), type I or type II diabetes, functional thyroid disease or thyroid cancer, preeclampsia, previous history of gestational diabetes, and obesity (body mass index, BMI, greater than or equal to $30 \mathrm{~kg} / \mathrm{m}^{2}$ ). 
After matching and investigating the inclusion criteria, adiponectin levels and $75 \mathrm{~g}$ glucose test were checked in 37 pregnant women with gestational diabetes and 37 non-diabetic pregnant women. Laboratory measurements of this design were performed in the central laboratory of Sari Imam Hospital. Then both case and control groups were followed up for 6 weeks after termination of pregnancy. Eventually, 6 weeks after termination of pregnancy, the women in both groups were evaluated for impaired glucose tolerance and metabolic syndrome according to the most recent criteria for metabolic syndrome.

NCEP ATPIII criteria were used to assess metabolic syndrome, and if at least three criteria were present, a person would be diagnosed with metabolic syndrome.

Diagnosis of gestational diabetes with oral glucose tolerance test (GTT). This test was performed with $75 \mathrm{~g}$ oral glucose in three stages: a) fasting; b) $1 \mathrm{~h}$ after taking $75 \mathrm{~g}$ of glucose; and c) $2 \mathrm{~h}$ after taking $75 \mathrm{~g}$ of glucose. This test should be performed in the morning after overnight fasting (at least 8 and at most $14 \mathrm{~h}$ ) followed by at least 3 days of unrestricted diet (equal to or greater than $150 \mathrm{~g}$ of carbohydrate per day) and activity. The patient should be in a sitting position. To make a diagnosis of 1 or more, intravenous glucose levels have to be at or above the specified values: $\mathrm{FBS}>=92 \mathrm{mg} / \mathrm{dl}$, $2 \mathrm{hpp}>=153 \mathrm{mg} / \mathrm{dl}$, and $1 \mathrm{hpp}>=180 \mathrm{mg} / \mathrm{dl}$. Screening tests were performed in week 24 to 28 of gestation. Adiponectin levels were also assessed using ELISA (Mediagnost co., Germany) method based on the manufacturer's instructions. Optical absorption at $405 \mathrm{~nm}$ was read using an ELISA reader (Stat Fax 3200, USA). Fasting blood glucose testing was performed enzymatically using MINDRAY device from China. Testing of triglycerides and HDL cholesterol was performed enzymatically using MINDRAY device from China. Qualitative control of the devices was performed at the beginning, during, and at the end of the experiments. Postpartum maternal weight was measured with the least clothes and no shoes and was recorded using digital scales with $100 \mathrm{~g}$ accuracy. Maternal height was measured using a measuring tape in standing position without shoes, while the shoulders were in normal condition. BMI was calculated by dividing the weight $(\mathrm{kg})$ by the square of the height $\left(\mathrm{m}^{2}\right)$. The size of the mother's waist in the thinnest area was assessed when the individual was at the end of natural exhalation. Measurements were made using an inflexible measuring tape without imposing any pressure on the individual body and with an accuracy of $1 \mathrm{~cm}$. All measurements were performed by one person in order to eliminate measurement error from different individuals. Blood pressure was measured from the right hand using standard methods by trained individuals $15 \mathrm{~min}$ after the study subjects were placed in sitting position and their arm was set at the heart level on a rigid support and then after a 15-min interval for the second time.

In line with the ethics and confidentiality of information of all study participants, the basics and objectives of the study, data confidentiality, and anonymity of checklists were declared. In addition, they could naturally refuse to participate. Mean and median indices along with $\mathrm{SD}$ and IQR were used to describe quantitative data. Frequency (percent) was also used to describe qualitative data. Appropriate statistical analysis was performed after Kolmogorov-Smirnov test. Accordingly, chi-square and Fisher tests were employed to compare the outcome ratios between the two groups. Comparisons were also made between the mean of quantitative variables with independent t-test or its non-parametric equivalent. Logistic regression was also used to calculate the odd's ratio when required. All statistical analyses of this study were performed using IBM SPSS version 21 software and $p$ value less than 0.05 was considered statistically significant.

\section{Results}

The mean age of pregnant women was $28.46 \pm 4.11$ years in the non-diabetic group and $30.03 \pm 4.71$ in the diabetic group. There was no statistical difference in the mean age of the two groups $(\mathrm{p}=0.123)$. The mean BMI of pregnant women was $25.46 \mathrm{~kg} / \mathrm{m}^{2}$ in the nondiabetic group and $25.67 \mathrm{~kg} / \mathrm{m}^{2}$ in the diabetic group, which did not indicate a significant statistical difference under $\mathrm{t}$-test $(\mathrm{p}=0.727)$.

In this study, pregnancy status was investigated in diabetic and non-diabetic pregnant women. The results are presented in Table 1, according to which the two groups were matched in terms of the number of pregnancies, the number of pregnancies over 20 weeks, and the number of abortions, live, or stillbirths with no significant difference between the two groups.

Based on diagnostic criteria, metabolic syndrome was determined and compared in pregnant women who suffered gestational diabetes and those who did not. The results of this comparison are presented in Table 2.

In this study, the mean serum adiponectin level in pregnant women was $8.35 \mathrm{ng} / \mathrm{ml}$ in the control group and $5.51 \mathrm{ng} / \mathrm{ml}$ in the intervention group 
Table 1

Pregnancy status among diabetic and non-diabetic pregnant women.

\begin{tabular}{|c|c|c|c|c|c|c|}
\hline \multirow{2}{*}{ Variable } & \multirow{2}{*}{ Number } & \multicolumn{2}{|c|}{ Normal GTT } & \multicolumn{2}{|c|}{ Impaired GTT } & \multirow{2}{*}{ p-value } \\
\hline & & Frequency & Percentage & Frequency & Percentage & \\
\hline \multirow{4}{*}{ Total number of pregnancies } & 1 & 11 & 29.7 & 14 & 37.8 & \multirow{4}{*}{0.842} \\
\hline & 2 & 14 & 37.8 & 11 & 29.7 & \\
\hline & 3 & 9 & 24.3 & 10 & 27.0 & \\
\hline & 4 & 3 & 8.1 & 2 & 5.4 & \\
\hline \multirow{3}{*}{ Number of pregnancies over 20 weeks } & 0 & 16 & 43.2 & 16 & 43.2 & \multirow{3}{*}{1.000} \\
\hline & 1 & 15 & 40.5 & 15 & 40.5 & \\
\hline & 2 & 6 & 16.2 & 6 & 16.2 & \\
\hline \multirow{3}{*}{ Number of live children } & 0 & 18 & 48.6 & 18 & 48.6 & \multirow{3}{*}{1.000} \\
\hline & 1 & 13 & 35.1 & 13 & 35.1 & \\
\hline & 2 & 6 & 16.2 & 6 & 16.2 & \\
\hline \multirow{3}{*}{ Number of previous abortion cases } & 0 & 25 & 67.6 & 28 & 75.7 & \multirow{3}{*}{0.433} \\
\hline & 1 & 12 & 32.4 & 8 & 21.6 & \\
\hline & 2 & 0 & 0.0 & 1 & 2.7 & \\
\hline \multirow{2}{*}{ History of fetal death over 20 weeks } & 0 & 37 & 100.0 & 36 & 97.3 & \multirow{2}{*}{0.314} \\
\hline & 1 & 0 & 0.0 & 1 & 2.7 & \\
\hline
\end{tabular}

Abbreviations: GTT - glucose tolerance test

Table2

Determination and comparison of metabolic syndrome in pregnant women with gestational diabetes and non-gestational diabetes.

\begin{tabular}{lcccccc}
\hline & & \multicolumn{2}{c}{ Normal GTT } & \multicolumn{2}{c}{ Impaired GTT } \\
\cline { 3 - 5 } & & Frequency & Percentage & Frequency & Percentage \\
\hline Metabolic & Yes & 7 & 18.9 & 8 & 21.6 & 0.314 \\
Syndrome & No & 30 & 81.1 & 29 & 78.4 & 0.4 \\
\hline
\end{tabular}

Abbreviations: GTT - glucose tolerance test

and there was a statistically significant difference between the mean levels of serum adiponectin under $t$-test $(p=0.003)$. Also, the correlation between serum adiponectin level in pregnant women with gestational diabetes and healthy pregnant women was investigated. The results of this study showed that there was a significant relationship between the level of adiponectin and impaired blood glucose in the negative direction $(r=-0.325$ and $p=0.006)$, but there was no significant relationship between serum adiponectin and normal blood glucose $(\mathrm{r}=0.360$ and $\mathrm{p}=0.400)$.

In this study, the correlation between maternal biometric indices and serum adiponectin levels was investigated in general and separately in pregnant women with and without gestational diabetes. The results are presented in Table 3, according to which there was no correlation between serum adiponectin level in pregnant women having normal GTT with maternal age and BMI. A similar evaluation of the women with impaired GTT indicated no correlation between serum adiponectin level with maternal age and BMI.

\section{Discussion}

The purpose of this study was to investigate the relationship between the serum adiponectin levels and the postpartum gestational diabetes. All participants were divided into two groups of 37 diabetic and non-diabetic women. There was no statistically significant difference regarding the mean age and BMI between the two groups. Mean serum adiponectin levels in non-diabetic pregnant women were significantly higher than those with gestational diabetes. In pregnant women with gestational diabetes, serum adiponectin level did not correlate with maternal age and BMI. Serum adiponectin level did not correlate with postpartum metabolic 
syndrome in the two groups. There was a positive correlation between serum adiponectin level and maternal gestational diabetes.

Pala et al. (2015) have conducted a descriptive study on the serum adiponectin levels in pregnant women with and without gestational diabetes at 24-28 weeks of gestation, at delivery (maternal and cord blood), and $24 \mathrm{~h}$ postpartum. Mean serum adiponectin levels were significantly lower in women with gestational diabetes than in women without impaired glucose tolerance. Serum adiponectin levels were significantly lower in the gestational diabetes group at the time of delivery than in the group without gestational diabetes. They concluded that serum adiponectin levels in pregnant women were altered by changes in glucose and insulin metabolism, and a decrease in adiponectin levels would reflect insulin resistance in gestational diabetes. Also, the mean serum adiponectin levels in pregnant women with diabetes were significantly lower than pregnant women without gestational diabetes. The study by Pala et al. (2015) have only examined the relationship between adiponectin levels and gestational diabetes and did not address other aspects of adiponectin; however, the present study has examined the association between gestational diabetes and adiponectin levels, after which the correlation of adiponectin levels and postpartum metabolic syndrome has been also investigated.

Ryckman et al. (2015) have conducted a metaanalysis study to investigate the blood lipid levels in mothers with and without gestational diabetes and concluded that pregnant women with gestational diabetes were at risk for postpartum metabolic syndrome. In the present study, there was no significant relationship between gestational diabetes and postpartum metabolic syndrome. In Rickman's study, risk factors such as smoking, previous history of gestational diabetes, or family history of diabetes and BMI were not considered and lipid blood levels were checked during the second and third trimesters of pregnancy. Therefore, considering the differences in inclusion criteria and lack of matching for these important risk factors which influence the metabolic syndrome and also regarding time interval differences in checking lipid levels, the differences in the results of the present study and Rickman's can be justified (Ryckman et al. 2015).

In the study by Bhograj et al. (2016), which aimed to correlate serum adiponectin levels with gestational diabetes, it has been found that adiponectin serum levels in diabetic pregnant women were significantly lower than the normal pregnancy group, which
Table 3

The correlation between maternal biometric indices and serum adiponectin levels.

\begin{tabular}{llcc}
\hline \multirow{2}{*}{ Variables } & \multirow{2}{*}{ Indexes } & \multicolumn{2}{c}{ Diabetes status } \\
\cline { 3 - 4 } & & Normal GTT & Impaired GTT \\
\hline Age & Count & 37 & 37 \\
& $\mathrm{r}$ & 0.111 & -0.087 \\
& $\mathrm{p}$-value & 0.514 & 0.609 \\
BMI & Count & 37 & 37 \\
& $\mathrm{r}$ & -0.284 & -0.212 \\
& $\mathrm{p}$-value & 0.088 & 0.207 \\
Metabolic & Count & 37 & 37 \\
Syndrome & $\mathrm{r}$ & 0.24 & 0.423 \\
& $\mathrm{p}$-value & 0.123 & 0.23 \\
\hline
\end{tabular}

Abbreviations: GTT - glucose tolerance test

is consistent with the results of the present study. Bhograj et al. (2016) have reported an average adiponectin level of $19.38 \mathrm{ng} / \mathrm{ml}$ in the normal group and $16.92 \mathrm{ng} / \mathrm{ml}$ in the diabetes group, which is consistent with our study. However, there is a difference in adiponectin levels of the entire population with the study of Bhograj et al. (2016), which may be related to racial and ethnic differences. It is important to note that environmental factors affect adiponectin levels and further research is required in this regard (Bhograj et al. 2016).

Saucedo et al. (2017) have indicated in their study that adiponectin serum levels in diabetic pregnant women were significantly lower than those in healthy pregnant women, which is consistent with the results of the present study. The study of Ianniello et al. (2013), which have been conducted as a prospective cohort study, showed that in each of the three trimesters in the gestational diabetes group, serum adiponectin levels were significantly lower than the control group. Accordingly, adiponectin, adipose mass, diastolic blood pressure, and HOMA were identified as dependent predictors of adiponectin. They concluded that pregnant women who developed gestational diabetes in the future had lower adiponectin serum levels than women with normal blood sugar. They also had higher body fat and higher blood pressure in the first trimester than women with normal blood glucose levels. Adiponectin, diastolic blood pressure, HOMA, and body fat are all dependent predictors of gestational diabetes, and adiponectin marker can be used to predict gestational diabetes in the future.

In the study of Hossein-Nezhad et al. (2010), the predictive effect of adiponectins in prediction of gestational diabetes and postpartum metabolic syndrome was investigated. They found that there was a signifi- 
cant difference between the three adipokine concentrations (adiponectin, visfantine, resistin) in pregnant women with diabetes mellitus and those with normal pregnancy. There was a significant relationship between the incidence of postpartum metabolic syndrome and low adiponectin serum levels during pregnancy. In the present study, serum adiponectin levels were significantly lower in diabetic pregnant women than in the non-diabetic group. The study concluded that there was a possible association between adiponectin concentrations and gestational diabetes. Also, serum adiponectin levels were not correlated with postpartum metabolic syndrome, which confirmed the results of Hossein-Nezhad et al. (2010).

In a systematic review and meta-analysis, Iliodromiti et al. (2016) have found that adiponectin circulation had $64.7 \%$ sensitivity and $77.8 \%$ specificity for predicting gestational diabetes mellitus. They concluded that measurements of adiponectin before pregnancy and at early pregnancy can be useful to identify women at high risk of gestational diabetes mellitus (Iliodromiti et al. 2016). Future evaluations can meet the need to GTT through combination of adiponectin and maternal findings, so that early diagnosis could be made.

In conclusion, the results of the present study indicate the association of adiponectin concentrations with gestational diabetes, but this association with postpartum metabolic syndrome is uncertain. However, to elucidate the mechanism of adiponectin in predicting gestational diabetes and postpartum metabolic syndrome further studies are required.

\section{Acknowledgments}

The study was approved by the ethics committee of Mazandaran University of Medical Sciences (grant number: 97/68). We would like to thank all the people who helped us with collecting the data and the searching works.

\section{References}

Ben-Haroush A, Yogev Y, Hod M. Epidemiology of gestational diabetes mellitus and its association with type 2 diabetes. Diabet Med 21, 103-113, 2004.

Bentley-Lewis R, Levkoff S, Stuebe A, Seely EW. Gestational diabetes mellitus: postpartum opportunities for the diagnosis and prevention of type 2 diabetes mellitus. Nat Clin Pract Endocrinol Metab 4, 552-558, 2008.

Bhograj A, Suryanarayana KM, Nayak A, Murthy NS, Dharmalingam M, Kalra P. Serum adiponectin levels in gestational diabetes mellitus. Indian J Endocrinol Metab 20, 752-755, 2016.

Daviglus ML, Stamler J, Pirzada A, Yan LL, Garside DB, Liu K, Wang R, Dyer AR, Lloyd-Jones DM, Greenland P. Favorable cardiovascular risk profile in young women and long-term risk of cardiovascular and all-cause mortality. JAMA 292, 1588-1592, 2004.

Di Cianni G, Miccoli R, Volpe L, Lencioni C, Del Prato S. Intermediate metabolism in normal pregnancy and in gestational diabetes. Diabetes Metab Res Rev 19, 259-270, 2003.

Fuglsang J, Skjaerbaek C, Frystyk J, Flyvbjerg A, Ovesen P. A longitudinal study of serum adiponectin during normal pregnancy. BJOG 113, 110-113, 2006.

Gonzalez Gonzalez NL, Gonzalez Davila E, Castro A, Padron E, Plasencia W. Effect of pregestational diabetes mellitus on first trimester placental characteristics: three-dimensional placental volume and power Doppler indices. Placenta 35, 147-151, 2014.

Hedderson MM, Darbinian J, Havel PJ, Quesenberry CP, Sridhar S, Ehrlich S, Ferrara A. Low prepregnancy adiponectin concentrations are associated with a marked increase in risk for development of gestational diabetes mellitus. Diabetes Care 36, 3930-3937, 2013.

Hossein-Nezhad, A, Mirzaei K, Maghbooli Z, Rahmani M, Larijani B. Resistin, adiponectin and visfatin; can adipocytokines predict gestational diabetes mellitus and early post partum metabolic syndrome? Iranian Journal of Diabetes and Lipid Disorders 9, 1-8, 2010.

Ianniello F, Quagliozzi L, Caruso A, Paradisi G. Low adiponectin in overweight/obese women: association with diabetes during pregnancy. Eur Rev Med Pharmacol Sci 17, 3197-3205, 2013.

Iliodromiti S, Sassarini J, Kelsey TW, Lindsay RS, Sattar N, Nelson SM. Accuracy of circulating adiponectin for predicting gestational diabetes: a systematic review and meta-analysis. Diabetologia 59, 692-699, 2016.

Innes KE, Wimsatt JH. Pregnancy-induced hypertension and insulin resistance, evidence for a connection. Acta Obstet Gynecol Scand 78, 263-284, 1999.

Kaufmann RC, Schleyhahn FT, Huffman DG, Amankwah KS. Gestational diabetes diagnostic criteria: long-term maternal follow-up. Am J Obstet Gynecol 172, 621-625, 1995. 
Khong SL, Kane SC, Brennecke SP, da Silva Costa F. First-trimester uterine artery Doppler analysis in the prediction of later pregnancy complications. Dis Markers 2015, 679730, 2015.

Kim C, Newton KM, Knopp RH. Gestational diabetes and the incidence of type 2 diabetes: a systematic review. Diabetes Care 25, 1862-1868, 2002.

Maruotti GM, Rizzo G, Sirico A, Sarno L, Cirigliano L, Arduini D, Martinelli P. Are there any relationships between umbilical artery Pulsatility Index and macrosomia in fetuses of type I diabetic mothers? J Matern Fetal Neonatal Med 27, 1776-1781, 2014.

Mastorakos G, Valsamakis G, Papatheodorou DC, Barlas I, Margeli A, Boutsiadis A, Kouskouni E, Vitoratos N, Papadimitriou A, Papassotiriou I, Creatsas G. The role of adipocytokines in insulin resistance in normal pregnancy: visfatin concentrations in early pregnancy predict insulin sensitivity. Clin Chem 53, 1477-1483, 2007.

Mazaki-Tovi S, Kanety H, Pariente C, Hemi R, Wiser A, Schiff E, Sivan E. Maternal serum adiponectin levels during human pregnancy. J Perinatol 27, 77-81, 2007.

Metzger BE, Cho NH, Roston SM, Radvany R. Prepregnancy weight and antepartum insulin secretion predict glucose tolerance five years after gestational diabetes mellitus. Diabetes Care 16, 1598-1605, 1993.

Miller JL, de Veciana M, Turan S, Kush M, Manogura A, Harman CR, Baschat AA. First-trimester detection of fetal anomalies in pregestational diabetes using nuchal translucency, ductus venosus Doppler, and maternal glycosylated hemoglobin. Am J Obstet Gynecol 208, 385.e1-385.e8, 2013.

Ouchi N, Kihara S, Arita Y, Okamoto Y, Maeda K, Kuriyama H, Hotta K, Nishida M, Takahashi M, Muraguchi M, Ohmoto Y, Nakamura T, Yamashita S, Funahashi T, Matsuzawa Y. Adiponectin, an adipocyte-derived plasma protein, inhibits endothelial NF- $\kappa B$ signaling through a cAMP-dependent pathway. Circulation 102, 1296-1301, 2000.

Pala HG, Ozalp Y, Yener AS, Gerceklioglu G, Uysal S, Onvural A. Adiponectin levels in gestational diabetes mellitus and in pregnant women without glucose intolerance. Adv Clin Exp Med 24, 85-92, 2015.

Retnakaran R, Hanley AJ, Raif N, Connelly PW, Sermer M, Zinman B. Hypoadiponectinaemia in South Asian women during pregnancy: evidence of ethnic variation in adiponectin concentration. Diabet Med 21, 388-392, 2004.Ryckman KK, Spracklen CN, Smith CJ, Robinson JG, Saftlas AF. Maternal lipid levels during pregnancy and gestational diabetes: a systematic review and meta-analysis. BJOG 122, 643-651, 2015.

Saucedo R, Valencia J, Gutierrez C, Basurto L, Hernandez M, Puello E, Rico G, Vega G, Zarate A. Gene variants in the FTO gene are associated with adiponectin and TNF-alpha levels in gestational diabetes mellitus. Diabetol Metab Syndr 9, 32, 2017.

Strehlow SL, Mestman JH. Prevention of T2DM in women with a previous history of GDM. Curr Diab Rep 5, 272$277,2005$. 